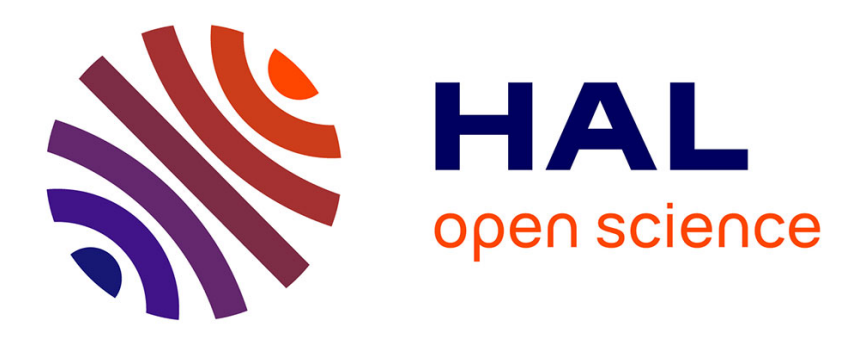

\title{
Theory of elasticity and consolidation for a porous anisotropic solid \\ Maurice A. Biot
}

\section{To cite this version:}

Maurice A. Biot. Theory of elasticity and consolidation for a porous anisotropic solid. Journal of Applied Physics, 1955, 26 (2), pp.182-185. 10.1063/1.1721956 . hal-01368659

\section{HAL Id: hal-01368659 \\ https://hal.science/hal-01368659}

Submitted on 21 Sep 2016

HAL is a multi-disciplinary open access archive for the deposit and dissemination of scientific research documents, whether they are published or not. The documents may come from teaching and research institutions in France or abroad, or from public or private research centers.
L'archive ouverte pluridisciplinaire HAL, est destinée au dépôt et à la diffusion de documents scientifiques de niveau recherche, publiés ou non, émanant des établissements d'enseignement et de recherche français ou étrangers, des laboratoires publics ou privés. 


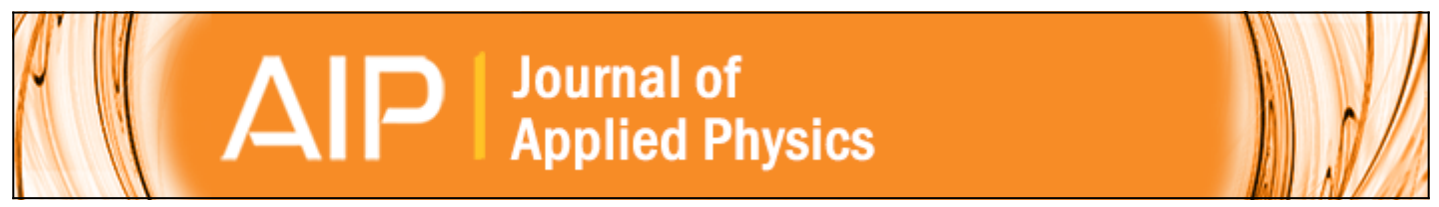

\section{Theory of Elasticity and Consolidation for a Porous Anisotropic Solid}

M. A. Biot

Citation: Journal of Applied Physics 26, 182 (1955); doi: 10.1063/1.1721956

View online: http://dx.doi.org/10.1063/1.1721956

View Table of Contents: http://scitation.aip.org/content/aip/journal/jap/26/2?ver=pdfcov

Published by the AIP Publishing

\section{Articles you may be interested in}

Theory of compressional and transverse wave propagation in consolidated porous media

J. Acoust. Soc. Am. 106, 575 (1999); 10.1121/1.427026

Creep Theory of Anisotropic Solids

J. Rheol. 25, 565 (1981); 10.1122/1.549631

Theory of Elasticity of an Anisotropic Elastic Body

Phys. Today 17, 84 (1964); 10.1063/1.3051394

Theory of Deformation of a Porous Viscoelastic Anisotropic Solid J. Appl. Phys. 27, 459 (1956); 10.1063/1.1722402

Theory of Propagation of Elastic Waves in a Fluid-Saturated Porous Solid. I. Low-Frequency Range J. Acoust. Soc. Am. 28, 168 (1956); 10.1121/1.1908239

\section{High Energy Nanosecond Lasers}

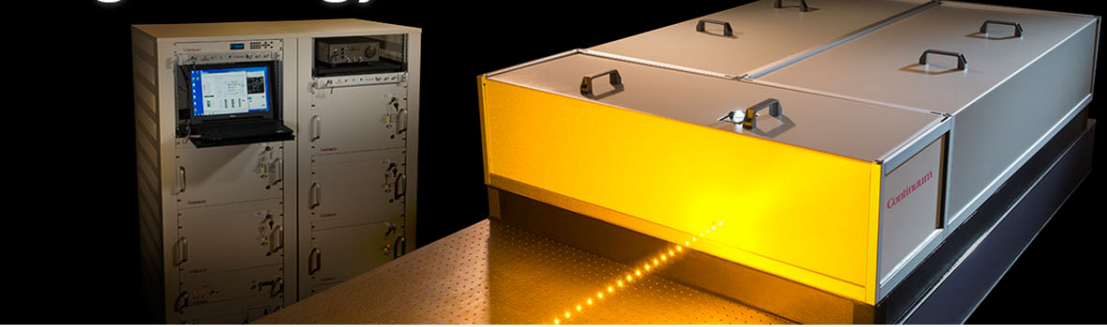

- Energies to $1 \mathrm{~kJ}$ - Variable Pulsewidths - Intuitive GUI for system control 


\title{
Theory of Elasticity and Consolidation for a Porous Anisotropic Solid
}

\author{
M. A. Brot* \\ Shell Development Company, New York City, New York
}

(Received May 5, 1954)

\begin{abstract}
The author's previous theory of elasticity and consolidation for isotropic materials []. Appl. Phys. 12, 155-164 (1941)] is extended to the general case of anisotropy. The method of derivation is also different and more direct. The particular cases of transverse isotropy and complete isotropy are discussed.
\end{abstract}

\section{INTRODUCTION}

$T$ HE theory of consolidation deals with the settlement under loading of a porous deformable solid containing a viscous fluid. In a previous publication ${ }^{1}$ a consolidation theory was developed for isotropic materials. The purpose of the present paper is to extend the theory to the most general case of anisotropy. The method by which the theory is derived is also more general and direct. The same physical assumption is introduced, that the skeleton is purely elastic and contains a compressible viscous fluid. The theory may therefore also be considered as a generalization of the theory of elasticity to porous materials. It is applicable to the prediction of the time history of stress and strain in a porous solid in which fluid seepage occurs. The general equations derived in Sec. 2 are applied to the case of transverse isotropy in Sec. 3. This is a case of particular interest in the application of the theory to soils and natural rock formations, since transverse isotropic is the type of symmetry usually acquired by rock under the influence of gravity. For an isotropic material the equations reduce to a simple form given in Sec. 4. They are shown to coincide with the equations derived in reference 1 . Application of the theory to specific cases was made previously, ${ }^{2-4}$ and it was shown that the operational calculus offers a very powerful tool for the solution of consolidation problems in which a load is applied to the material at a given instant and the time history of the settlement is to be calculated. These methods are directly applicable to the more general nonisotropic case. More general solutions of the equations have been developed and will be presented in a forthcoming publication.

\section{GENERAL EQUATIONS FOR THE ANISOTROPIC CASE}

Let us consider an elastic skeleton with a statistical distribution of interconnected pores. This porosity is usually denoted by

$$
f=V_{p} / V_{b},
$$

where $V_{p}$ is the volume of the pores contained in a

* Consultant

1 M. A. Biot, J. Appl. Phys. 12,155-164 (1941).

2 M. A. Biot, J. Appl. Phys, 12, 426-430 (1941),

M. A. Biot and F. M. Clingan, J. Appl. Phys. 12, 578-581 (1941).

4 M. A. Biot and F. M. Clingan, J. Appl. Phys. 13, 35-40 (1942). sample of bulk volume $V_{b}$. It is understood that the term "porosity" refers as is customary to the effective porosity, namely, that encompassing only the intercommunicating void spaces as opposed to those pores which are sealed off. In the following, the word "pore" will refer to the effective pores while the sealed pores will be considered as part of the solid. It will be noted that a property of the porosity $f$ is that it represents also a ratio of areas

$$
f=S_{p} / S_{b},
$$

i.e., the fraction $S_{p}$ occupied by the pores in any crosssectional area $S_{b}$ of the bulk material. It must beassumed, of course, that the pores are randomly distributed in location but not necessarily in direction. That this relation holds may be ascertained by integrating $S_{p} / S_{b}$ over a length of unity in a direction normal to the cross section $S_{b}$. The value of this integral then represents the fraction $f$ of the volume occupied by the pores. It is seen that the ratio $S_{p} / S_{b}$ is also independent of the direction of the cross section.

The stress tensor in the porous material is

$$
\left\{\begin{array}{ccc}
\sigma_{x x}+\sigma & \sigma_{x y} & \sigma_{x z} \\
\sigma_{y x} & \sigma_{y y}+\sigma & \sigma_{y z} \\
\sigma_{z x} & \sigma_{z y} & \sigma_{z z}+\sigma
\end{array}\right\},
$$

with the symmetry property $\sigma_{i j}=\sigma_{j i}$.

The partial components of this tensor do not have the conventional significance. If we consider a cube of unit size of the bulk material, $\sigma$ represents the total normal tension force applied to the fluid part of the faces of the cube. Denoting by $p$ the hydrostatic pressure of the fluid in the pores we may write

$$
\sigma=-f p
$$

The remaining components $\sigma_{x x}, \sigma_{x y}$, etc., of the tensor are the forces applied to that portion of the cube faces occupied by the solid.

We shall now call our attention to this system of fluid and solid as a general elastic system with conservation properties. The solid skeleton is considered to have compressibility and shearing rigidity, and the fluid may be compressible. The deformation of a unit cube is assumed to be completely reversible. By deformation is meant here that determined by both strain tensors in the solid and the fluid which will now be defined. The average displacement components of the solid is designated by $u_{x}, u_{y}, u_{z}$, and that of the fluid by $U_{x}, U_{y}, U_{z}$. 
The strain components for the solid and the fluid, respectively, are

$$
\begin{array}{lll}
e_{x x}=\frac{\partial u_{x}}{\partial x} \quad \frac{1}{2} e_{x y}=\frac{1}{2} & \left(\frac{\partial u_{y}}{\partial x}+\frac{\partial u_{x}}{\partial y}\right) \text { etc. } \\
\epsilon_{x x}=\frac{\partial U_{x}}{\partial x} \quad \frac{1}{2} \epsilon_{x y}=\frac{1}{2}\left(\frac{\partial U_{y}}{\partial x}+\frac{\partial U_{x}}{\partial y}\right) \text { etc. }
\end{array}
$$

By a generalization of the procedure followed in the classical theory of elasticity (5) we may write for the elastic potential energy $V$ the expression

$$
\begin{aligned}
2 V=\sigma_{x x} e_{x x}+\sigma_{y y} e_{y y}+\sigma_{z z} e_{z z} & +\sigma_{y z} e_{y z} \\
& +\sigma_{z x} e_{z x}+\sigma_{x y} e_{x y}+\sigma \epsilon
\end{aligned}
$$

with

$$
\epsilon=\epsilon_{x x}+\epsilon_{y y}+\epsilon_{z z}
$$

If we assume that the seven stress components are linear functions of the seven strain components the expression $2 \mathrm{~V}$ is a homogeneous quadratic function of the strain. This function is a positive definite form with twenty-eight distinct coefficients. The stress components are given by the partial derivatives of $V$ as follows:

$$
\begin{aligned}
\partial V / \partial e_{x x} & =\sigma_{x x} \quad \partial V / \partial e_{x y}=\sigma_{x y}, \quad \text { etc. }, \\
\partial V / \partial \epsilon & =\sigma .
\end{aligned}
$$

This is written

$$
\left(\begin{array}{l}
\sigma_{z x} \\
\sigma_{z y} \\
\sigma_{z z} \\
\sigma_{y z} \\
\sigma_{z x} \\
\sigma_{z y} \\
\sigma
\end{array}\right)=\left(\begin{array}{r}
c_{11} c_{12} c_{13} c_{14} c_{15} c_{16} c_{17} \\
c_{22} c_{23} c_{24} c_{25} c_{26} c_{27} \\
c_{33} c_{34} c_{35} c_{36} c_{37} \\
c_{44} c_{45} c_{46} c_{47} \\
c_{55} c_{56} c_{57} \\
c_{66} c_{67} \\
c_{77}
\end{array}\right)\left(\begin{array}{l}
e_{z x} \\
e_{z y} \\
e_{z z} \\
e_{y z} \\
e_{z x} \\
e_{x y} \\
\epsilon
\end{array}\right) .
$$

Because the matrix of coefficients is that of a quadratic form we have the symmetry property

$$
c_{i j}=c_{j i} \text {. }
$$

The total stress field (2.3) of the bulk material satisfies the equilibrium equations

$$
\begin{aligned}
& \frac{\partial}{\partial x}\left(\sigma_{x x}+\sigma\right)+\frac{\partial \sigma_{x y}}{\partial y}+\frac{\partial \sigma_{x z}}{\partial z}+\rho X=0 \\
& \frac{\partial \sigma_{y x}}{\partial x}+\frac{\partial}{\partial y}\left(\sigma_{y y}+\sigma\right)+\frac{\partial \sigma_{y z}}{\partial z}+\rho Y=0 \\
& \frac{\partial \sigma_{z x}}{\partial x}+\frac{\partial \sigma_{z y}}{\partial y}+\frac{\partial}{\partial z}\left(\sigma_{z z}+\sigma\right)+\rho Z=0
\end{aligned}
$$

where $\rho$ is the mass density of the bulk material and $X, Y, Z$, the body force per unit mass. Substituting in (2.10) the stress components as functions of the strains from (2.8) we obtain three equations for the six unknown displacement $u_{x} \cdots U_{x} \cdots$. Three further equa- tions between these unknowns are obtained by introducing the law governing the flow of a fluid in a porous material.

We introduce here a generalized form of Darcy's law for a nonisotropic material

$$
\left[\begin{array}{l}
-\partial p / \partial x+\rho_{f} X \\
-\partial p / \partial y+\rho_{f} Y \\
-\partial p / \partial z+\rho_{f} Z
\end{array}\right]=\left[\begin{array}{lll}
k_{x x} & k_{x y} & k_{z z} \\
k_{y x} & k_{y y} & k_{y z} \\
k_{z x} & k_{z y} & k_{z z}
\end{array}\right]\left[\begin{array}{l}
\dot{U}_{x}-\dot{u}_{x} \\
\dot{U}_{y}-\dot{u}_{y} \\
\dot{U}_{z}-\dot{u}_{z}
\end{array}\right],
$$

where $\rho_{f}$ is the mass density of the fluid. The matrix $k_{i j}$ constitutes a generalization of Darcy's constant if we include in it the viscosity coefficient. The average velocities of the fluid and solid are denoted by $\dot{U}_{x} \cdots \dot{u}_{x} \cdots$

The symmetry of the coefficients

$$
k_{i j}=k_{j i}
$$

results from the existence of a dissipation function such that the rate of dissipation of the energy in the porous material at rest is expressed by the positive definite quadratic form

$$
2 D=\sum^{i j} k_{i j} \dot{U}_{i} \dot{U}_{j}
$$

If we multiply Eq. (2.11) by $f$ and take (2.4) into account we obtain

$$
\left.\left[\begin{array}{l}
\partial \sigma / \partial x+\rho_{1} X \\
\partial \sigma / \partial y+\rho_{1} Y \\
\partial \sigma / \partial z+\rho_{1} Z
\end{array}\right]=\left[\begin{array}{lll}
b_{x x} & b_{x y} & b_{x z} \\
b_{y x} & b_{y y} & b_{y z} \\
b_{z x} & b_{z y} & b_{z z}
\end{array}\right] \begin{array}{l}
\dot{U}_{x}-\dot{u}_{x} \\
\dot{U}_{y}-\dot{u}_{y} \\
\dot{U}_{z}-\dot{u}_{z}
\end{array}\right],
$$

with $\rho_{1}=\rho_{f} f=$ the mass of fluid per unit volume of bulk material. The three equations obtained by combining (2.10) and (2.8) in addition to the three Eqs. (2.14) determine the six unknown displacement components for the fluid and the solid.

\section{THE CASE OF TRANSVERSE ISOTROPY}

The above equations are valid for the most general case of a symmetry. In practice, however, materials will be either isotropic or exhibit a high degree of symmetry which greatly simplifies the equations. Let us consider first the case of a material which is axially symmetric about the $z$ axis. This type of symmetry is referred to by Love ${ }^{5}$ as transverse isotropy (page 160). The expression for the strain energy in this case is

$$
\begin{aligned}
2 V= & (A+2 N)\left(e_{x x}+e_{y y}\right)^{2}+C e_{z z}{ }^{2}+2 F\left(e_{y y}+e_{x x}\right) e_{z z} \\
& +L\left(e_{y z}{ }^{2}+e_{z x}{ }^{2}\right)+N\left(e_{x y}{ }^{2}-4 e_{x x} e_{y y}\right) \\
& +2 M\left(e_{x x}+e_{y y}\right) \epsilon+2 Q e_{z z} \epsilon+R \epsilon^{2} .
\end{aligned}
$$

This expression is invariant under a rotation arount the $z$ axis. It is written in such a way as to bring out expressions such as $e_{x y}{ }^{2}-4 e_{x x} e_{y y}$ and $e_{x x}+e_{y y}$ which are invariant under a rotation about the $z$ axis. The coefficient $A+2 N$ is written this way for reasons of conformity.

\footnotetext{
${ }^{5}$ A. E. H. Love, A Treatise On the Mathematical Theory of Elasticity (Dover Publications, New York, 1944).
} 
Since $A$ does not appear in any other term, the quantity $A+2 N$ is an independent coefficient which could have been written as $P$ [see (4.5)]. The stress-strain relations derived from (2.7) and (3.1) are

$$
\begin{aligned}
\sigma_{x x} & =2 N e_{x x}+A\left(e_{x x}+e_{y y}\right)+F e_{z z}+M \epsilon ; \\
\sigma_{y y} & =2 N e_{y y}+A\left(e_{x x}+e_{y y}\right)+F e_{z z}+M \epsilon ; \\
\sigma_{z z} & =C e_{z z}+F\left(e_{x x}+e_{y y}\right)+Q \epsilon ; \\
\sigma_{y z} & =L e_{y z} ; \\
\sigma_{z x} & =L e_{z x} ; \\
\sigma_{x y} & =N e_{x y} ; \\
\sigma & =M\left(e_{x x}+e_{y y}\right)+Q e_{z z}+R \epsilon .
\end{aligned}
$$

There are therefore in this case eight elastic coefficients.

The equations of flow contain two coefficients of permeability, one in the $z$ direction, the other in the $x, y$ plane, and may be written

$$
\begin{aligned}
& \partial \sigma / \partial x+\rho_{1} X=b_{x x}\left(\dot{U}_{x}-\dot{u}_{x}\right) \\
& \partial \sigma / \partial y+\rho_{1} Y=b_{x x}\left(\dot{U}_{y}-\dot{u}_{y}\right) \\
& \partial \sigma / \partial z+\rho_{1} Z=b_{z z}\left(\dot{U}_{z}-\dot{u}_{z}\right) .
\end{aligned}
$$

These equations along with the stress-strain relation (3.2) and the equilibrium relations (2.10) yield six equations for the six displacement components in the case of transverse isotropy.

\section{THE CASE OF ISOTROPY}

In the case of complete isotropy the strain energy function (3.1) becomes

$$
\begin{aligned}
2 V= & (A+2 N)\left(e_{x x}+e_{y y}+e_{z z}\right)^{2} \\
& +N\left(e_{y z}{ }^{2}+e_{z x}{ }^{2}+e_{x y}{ }^{2}-4 e_{y y} e_{z z}\right. \\
& \left.-4 e_{z z} e_{x x}-4 e_{x x} e_{y y}\right) \\
& +2 Q\left(e_{x x}+e_{y y}+e_{z z}\right) \epsilon+R \epsilon^{2}
\end{aligned}
$$

We put

$$
e=e_{x x}+e_{y y}+e_{z z} \text {. }
$$

The stress-strain relations derived from (2.7) are

$$
\begin{aligned}
\sigma_{x x} & =2 N e_{x x}+A e+Q \epsilon ; \\
\sigma_{y y} & =2 N e_{y y}+A e+Q \epsilon ; \\
\sigma_{z z} & =2 N e_{z z}+A e+Q \epsilon ; \\
\sigma_{y z} & =N e_{y z} ; \\
\sigma_{z x} & =N e_{z x} ; \\
\sigma_{x y} & =N e_{x y} ; \\
\sigma & =Q e+R \epsilon .
\end{aligned}
$$

There are in this case four elastic constants, and this checks with the result obtained in reference 1 . The equations of flow contain a single coefficient $b$. They are written

$$
\begin{aligned}
& \partial \sigma / \partial x+\rho_{1} X=b\left(\dot{U}_{x}-\dot{u}_{x}\right) \\
& \partial \sigma / \partial y+\rho_{1} Y=b\left(\dot{U}_{y}-\dot{u}_{y}\right) \\
& \partial \sigma / \partial z+\rho_{1} Z=b\left(\dot{U}_{z}-\dot{u}_{z}\right) .
\end{aligned}
$$

We shall assume that there is no body force and put $X=Y=Z=0$. Substitution of expression (4.3) into the equilibrium Eq. (2.10) for the stresses and the flow Eq. (4.4) yield the six equations

$$
\begin{aligned}
& N \nabla^{2} \bar{u}+(P-N+Q) \operatorname{grad} e+(Q+R) \operatorname{grad} \epsilon=0 \\
& \operatorname{grad}(Q e+R \epsilon)=b(\partial / \partial t)(\bar{U}-\bar{u}) .
\end{aligned}
$$

We have put $P=A+2 N$.

Taking the divergence of the second equation we may also write

$$
\begin{aligned}
& N \nabla^{2} \bar{u}+(P-N+Q) \operatorname{grad} e+(Q+R) \operatorname{grad} \epsilon=0 \\
& Q \nabla^{2} e+R \nabla^{2} \epsilon=b(\partial / \partial t)(\epsilon-e) .
\end{aligned}
$$

In the previous theory (1) we had obtained these equations by a different method and in a different form. To show their equivalence we write the stress-strain relations by eliminating $\epsilon$ from Eqs. (4.3)

$$
\begin{aligned}
& \sigma_{x x}=2 N e_{x x}+\left(A-\frac{Q^{2}}{R}\right) e+\frac{Q}{R} \sigma ; \\
& \sigma_{y y}=2 N e_{y y}+\left(A-\frac{Q^{2}}{R}\right) e+\frac{Q}{R} \sigma ; \\
& \sigma_{z z}=2 N e_{z z}+\left(A-\frac{Q^{2}}{R}\right) e+\frac{Q}{R} \sigma ; \\
& \sigma_{y z}=N e_{y z} ; \\
& \sigma_{z x}=N e_{z x} ; \\
& \sigma_{x y}=N e_{x y} .
\end{aligned}
$$

Substituting these in the equilibrium relation (2.10) we find

$N \nabla^{2} \bar{u}+\left[P-N-Q^{2} / R\right] \operatorname{grad} e$

$$
+(Q+R) / R \operatorname{grad} \sigma=0 .
$$

We also derive from (4.4)

$$
\nabla^{2} \sigma=b \frac{\partial}{\partial t}(\epsilon-e)=\frac{b}{R} \frac{\partial \sigma}{\partial t}-b \frac{Q+R}{R} \frac{\partial e}{\partial t} .
$$

Equations (4.8) and (4.9) are in the form obtained in reference 1 . We note that the significance of $\sigma$ in that reference is equivalent to $-\sigma / f$ in our present notation.

Consider now the case of an incompressible material. This corresponds to the condition

$$
e(1-f)+f \epsilon=0 .
$$

Since this must be satisfied for all values of $\sigma$ we derive from the last relation (4.3) that both $R$ and $Q$ are infinite with the condition

$$
Q / R=(1-f) / f .
$$

Since $A-Q^{2} / R=S$ must remain finite the stress strain 
law becomes

$$
\begin{aligned}
\sigma_{x x} & =2 N e_{x x}+S e+\frac{1-f}{f} \sigma ; \\
\sigma_{y y} & =2 N e_{y y}+S e+\frac{1-f}{f} \sigma \\
\sigma_{z z} & =2 N e_{z z}+S e+\frac{1-f}{f} \sigma \\
\sigma_{y z} & =N e_{y z} \\
\sigma_{z x} & =N e_{x x} \\
\sigma_{x y} & =N e_{x y} .
\end{aligned}
$$

Substituting these expressions in the equilibrium relations (2.10) we derive

$$
N \nabla^{2} \bar{u}+(N+S) \operatorname{grad} e+(1 / f) \operatorname{grad} \sigma=0
$$

and from (4.9)

$$
\nabla^{2} \sigma=-\frac{b \partial e}{f} \frac{\partial t}{.}
$$

Taking the divergence of (4.13)

$$
(2 N+S) \nabla^{2} e+\frac{1}{f} \nabla^{2} \sigma=0 .
$$

Hence (4.14) may be written

$$
f^{2}(2 N+S) \nabla^{2} e=b \frac{\partial e}{\partial t}
$$

This is the equation of heat conduction. Equations (4.13) and (4.16) coincide with those obtained in reference 1 for the incompressible case.

\title{
Radiation of Plasma Noise from Arc Discharge
}

\author{
T. Takakura, K. Baba, K. Nunogaki, and H. Mrtani \\ Osaka City University, Kitaku, Osaka, Japan
}

(Received June 8, 1954)*

\begin{abstract}
The mechanism of radiation of plasma noise inherent to arc discharge is studied experimentally. Intense electromagnetic radiation of random noise character is observed at the frequency of plasma oscillation of the ion sheath formed at the cathode drop. Maximum radiation occurs when the external circuit connected to the cathode is in resonance with the plasma frequency. The oscillating current in the external circuit is concluded to be generated by the periodic electron emission from the cathode caused by small perturbation of potential gradient at the cathode surface as a result of the variation in the ion sheath potential. This conclusion is supported by several experiments under various air pressures, electrode materials, current densities, etc.
\end{abstract}

\section{INTRODUCTION}

$\mathrm{C}$ OLD cathode $\mathrm{dc}$ arc discharge in air at ordinary atmospheric pressure was found to radiate intense radio noise in the microwave region equivalent to thermal radiation of $10^{6}$ to $10^{7}{ }^{\circ} \mathrm{K}$ at 0.5 to 5 amperes of discharge current without any external resonance circuit. The radiation was observed at frequencies of $3300 \mathrm{mc}, 190 \mathrm{mc}, 15 \mathrm{mc}$, and $1.5 \mathrm{mc}$ under various air pressures with various electrode materials and shapes by detecting the noise involved therein.

More or less similar investigations were conducted by Melloh and others. ${ }^{1}$ However, little seems to have been done in the explanation of the mechanism of noise radiation. The present paper deals with the explanation of the generation of such noise radiation.

\footnotetext{
* The original unrevised manuscript was received September 8 , 1953.

A. M. Melloh, Proc. Inst. Radio Engrs. 28, 179 (1940); I. Matsumoto, Oyố-Butsuri 15, 21 (1946); I. Hayashi and E. Abe, Rikoken-Hökoku 3, 9 (1949); Davies, Pear, and White, Electronics 23, 96 (1950).
}

\section{EXPERIMENTAL RESULTS}

\section{(i) The Noise of Arc Discharge at an Atmospheric Pressure}

Radiation of noise from $\mathrm{dc}$ arc discharge in air was observed by using a $3300 \mathrm{mc}$ receiver with a band width of approximately $4 \mathrm{mc}$ connecting to a horn antenna. The condition of discharge was changed by the shape and material of the electrodes and the discharge current. The radio noise radiated from the arc discharge was received by the horn antenna as shown in Fig. 1(a).

\section{(a) Influence of Electrode Material}

Relative intensity of radio noise at $3300 \mathrm{mc}$ from the $\mathrm{dc}$ arc discharge at 1 ampere with electrodes of various materials was measured and tabulated in Table I. Care was taken to make each electrode of different materials in a similar shape. As seen in Table I, the positive electrode was found to be of little significance in determining the intensity of the noise. When a carbon or mercury electrode was used as the cathode, an excep- 